\title{
Design and characterization of a thermal hydrogen atom source
}

K. G. Tschersich, J. P. Fleischhauer, and H. Schuler

Citation: Journal of Applied Physics 104, 034908 (2008);

View online: https://doi.org/10.1063/1.2963956

View Table of Contents: http://aip.scitation.org/toc/jap/104/3

Published by the American Institute of Physics

\section{Articles you may be interested in}

Intensity of a source of atomic hydrogen based on a hot capillary

Journal of Applied Physics 87, 2565 (2000); 10.1063/1.372220

Formation of an atomic hydrogen beam by a hot capillary

Journal of Applied Physics 84, 4065 (1998); 10.1063/1.368619

Simple source of atomic hydrogen for ultrahigh vacuum applications

Journal of Vacuum Science \& Technology A: Vacuum, Surfaces, and Films 11, 458 (1998); 10.1116/1.578754

Quantitative characterization of a highly effective atomic hydrogen doser

Journal of Vacuum Science \& Technology A: Vacuum, Surfaces, and Films 16, 2979 (1998); 10.1116/1.581449

Novel method for absolute quantification of the flux and angular distribution of a radical source for atomic hydrogen

Journal of Vacuum Science \& Technology A: Vacuum, Surfaces, and Films 18, 995 (2000); 10.1116/1.582289

On the thermal dissociation of hydrogen

Journal of Applied Physics 66, 5749 (1998); 10.1063/1.343643

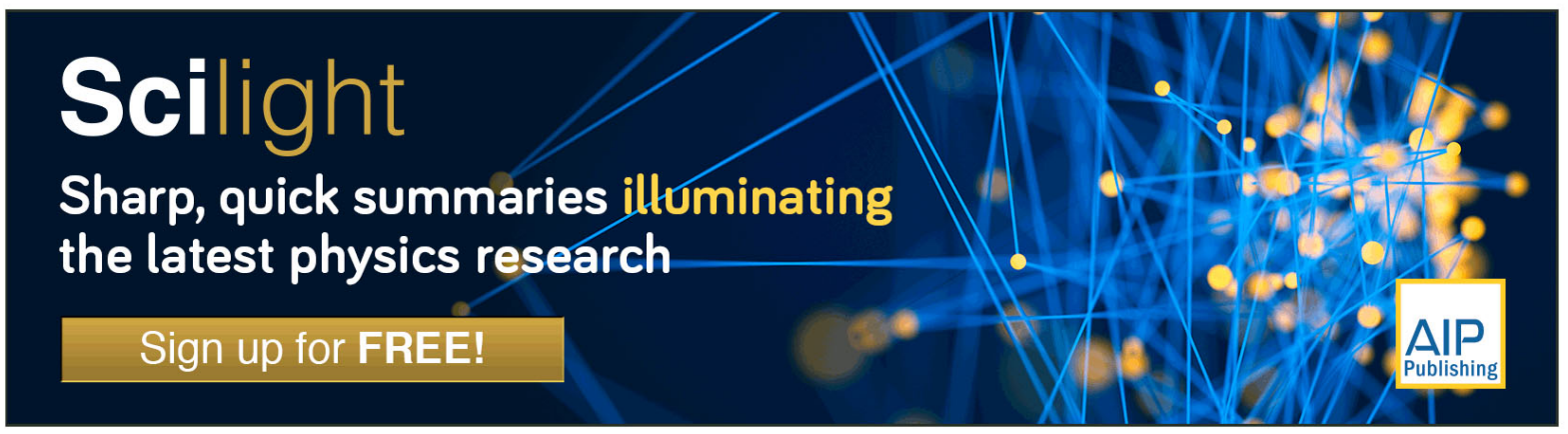




\title{
Design and characterization of a thermal hydrogen atom source
}

\author{
K. G. Tschersich, ${ }^{1, a)}$ J. P. Fleischhauer, ${ }^{1}$ and H. Schuler ${ }^{2}$ \\ ${ }^{1}$ Institut für Bio- und Nanosysteme-4, Forschungszentrum Jülich, D-52425 Jülich, Germany \\ ${ }^{2}$ MBE-Komponenten GmbH, Gutenbergstr. 8, D-71263 Weil der Stadt, Germany
}

(Received 9 January 2008; accepted 4 June 2008; published online 5 August 2008)

\begin{abstract}
The hydrogen atom source considered here incorporates a hot capillary fed by hydrogen gas. Our earlier measurements on a source heated by electron bombardment are interpreted in terms of a simple model which encourages us to design a source heated by the radiation from a filament. The radiatively heated source is much simpler, more reliable, and easier to run than the electronically heated source. Furthermore, the radiatively heated source is free of any energetic particles. In order to obtain quantitative data on the intensity, an apparatus is constructed revealing the angular distribution of the hydrogen atoms and molecules by means of a quadrupole mass analyzer. The intensity of the source is controlled by the mass flow rate of the feed gas and the electric power to the filament. The flux density of hydrogen atoms at a substrate $6 \mathrm{~cm}$ away from the source is variable over two orders of magnitude and extends up to some $10^{15}$ atoms $/ \mathrm{cm}^{2}$ s. (C) 2008 American Institute of Physics. [DOI: 10.1063/1.2963956]
\end{abstract}

\section{INTRODUCTION}

Hydrogen atoms find various applications in surface and thin film experiments. They are used to clean surfaces, e.g., of $\mathrm{Si}^{1}{ }^{1} \mathrm{GaAs},{ }^{2}$ and $\mathrm{Co},{ }^{3}$ and to cover surfaces in studies of adsorption, ${ }^{4}$ recombination, ${ }^{5,6}$ and desorption. ${ }^{7}$ In thin film deposition, hydrogen atoms are incorporated into the growing film. 8,9

Hydrogen atoms are predominantly produced by dissociation of hydrogen gas either in a plasma or at a hot surface. The latter has the advantage of providing hydrogen atoms free of any ions or excited molecules which may be detrimental to the substrate or obscure the pure chemical action of the atoms. There are two main versions of thermal hydrogen atom sources, i.e., the hot filament and the hot capillary source. The hot filament is run in an atmosphere of hydrogen gas, emits atoms in all directions, and burdens the vacuum pump with a rather high gas load. The hot capillary, in contrast, preferentially emits along the axis and has a comparatively low gas load.

Hydrogen atom sources incorporating a hot capillary and the work on their quantification were discussed in two earlier publications. $^{10,11}$ Meanwhile, Kim and Lee ${ }^{12}$ described a source similar to that of Bischler and Bertel. ${ }^{13}$ The atom flux at the sample surface was estimated from adsorption measurements using a Si surface. Horn et al. ${ }^{14}$ heated a capillary resistively by contacting a wire to the capillary near the orifice and passing a current through wire and capillary. Zecho et $a{ }^{15}{ }^{15}$ used this source for the etching of $a-\mathrm{Si}: \mathrm{H}$. They determined the angular distribution of the emitted atoms by the method of Schwarz-Selinger et al., ${ }^{16}$ where the spatial erosion of an exposed $a-\mathrm{C}: \mathrm{H}$ film in combination with the otherwise determined erosion yield reveals the hydrogen atom flux.

In previous work, ${ }^{10,11}$ we constructed and investigated a source of the hot capillary type. The capillary was heated by

${ }^{a)}$ Electronic mail: k.g.tschersich@fz-juelich.de. electron bombardment to make high temperatures accessible. Hydrogen atoms effusing from the orifice of the capillary were detected by a quadrupole mass analyzer (QMA) positioned in a part of the vacuum system which was differentially pumped. At the highest temperature to which the capillary was heated $(2600 \mathrm{~K})$ we measured the angular distribution of the hydrogen atoms in a limited polar angle range. The angular distribution was described by an analytic function $^{10}$ by means of which the measured data were extrapolated to the polar angle range $0 \leq \vartheta \leq 90^{\circ}$ of the hemisphere. We also measured the variation of the on-axis signal as a function of the capillary temperature and the gas flow through the capillary. From these data, i.e., the gas flow in absolute numbers and the angular distribution of the hydrogen atoms as well as their variation with temperature in relative numbers, we determined the intensity of the source. ${ }^{11}$

The present work goes beyond our previous investigations in two respects. (1) Based on insights into dissociation and beam formation in the capillary, we change from electron bombardment heating to heating by thermal radiation which makes the source much simpler, more reliable, and easier to run. For technical reasons as well as with respect to the vaporization of the construction material, we limit the temperature range of the capillary. This requires a more sensitive detection system. (2) We build an improved QMA apparatus which reveals the angular distribution of the atoms and also of the molecules at arbitrary temperature.

Sections II, III, and IV describe the design of the source, the QMA apparatus and its calibration, and the performance of the source in quantitative data.

\section{SOURCE DESIGN}

The previously measured angular distribution of the hydrogen atoms as well as the temperature dependence of their on-axis signal were both described by one single empirical parameter each. ${ }^{11}$ Accordingly, we can set up a simple model representing the dissociation and beam formation by the cap- 


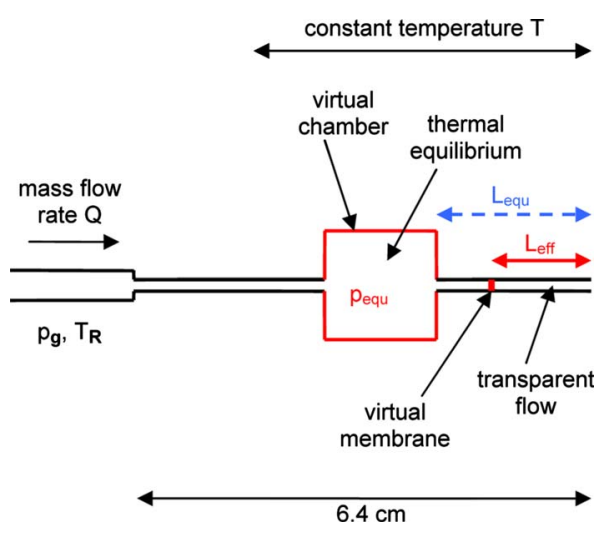

FIG. 1. (Color online) Model representing dissociation by means of the parameter $p_{\text {equ }}$ and beam formation by means of the parameter $L_{\text {eff. }} L_{\text {equ }}$ marks the position of the virtual chamber. The absolute length of the freestanding capillary is indicated. The gas duct is at pressure $p_{g}$ and at room temperature $T_{R}$.

illary, as shown in Fig. 1. We assume that the capillary is fed with hydrogen gas at a mass flow rate $Q$ and the greater part of the capillary up to the orifice is at temperature $T$. $Q$ and also $T$, which depends on the heating power, are variable experimental parameters. The dissociation can then be treated as if there were a virtual chamber in thermal equilibrium at $T$ and $p_{\text {equ }}$, where the pressure $p_{\text {equ }}$ is an empirical parameter depending on $Q$ only. [The temperature determines the equilibrium constant $K_{p}$, which in combination with $p_{\text {equ }}$ determines the degree of equilibrium dissociation $\alpha$ in the virtual chamber, see Eq. (7) in Ref. 10]. The angular distribution of the atoms leaving the orifice corresponds to that obtained by assuming a virtual membrane in the capillary and a transparent flow from the membrane up to the orifice. ${ }^{10}$ The length of the transparent flow is $L_{\mathrm{eff}}$, which is the other empirical parameter. $L_{\text {eff }}$ depends on $Q$ and $T$. (In Ref. 10 a dependence of $L_{\text {eff }}$ on $T$ was not taken into account since the angular distribution of hydrogen atoms was only measured at the highest temperature accessible. However, the dependence of $L_{\text {eff }}$ on $T$ became apparent in the present measurements.)

We wish to verify whether the relative positions of the virtual chamber and the virtual membrane are located, as sketched in Fig. 1. This requires an estimate of the distance $L_{\text {equ }}$ between the exit of the virtual chamber and the orifice. Accordingly, we have to discover the position at which the pressure in the capillary is equal to $p_{\text {equ }}$. The capillary temperature rises from about room temperature $T_{R}$ at the watercooled socket to the nominal temperature $T$, which is assumed to prevail up to the orifice. In that part of the capillary where the temperature rises, the pressure increases from the feeding pressure $p_{g}$ in the gas duct to a pressure of the order of $p_{g}\left(T / T_{R}\right)^{1 / 2}$ due to thermal transpiration. ${ }^{17,18}$ In the constant temperature part of the capillary, the pressure decreases to a very low level at the orifice. Let us now focus on that part of the capillary marked by $L_{\text {equ }}$. If we assume molecular flow, the pressure along $L_{\text {equ }}$ decreases linearly and the gas flow rate $Q_{T}$ is related to the molecular flow conductance $C_{\text {mol }}$ by

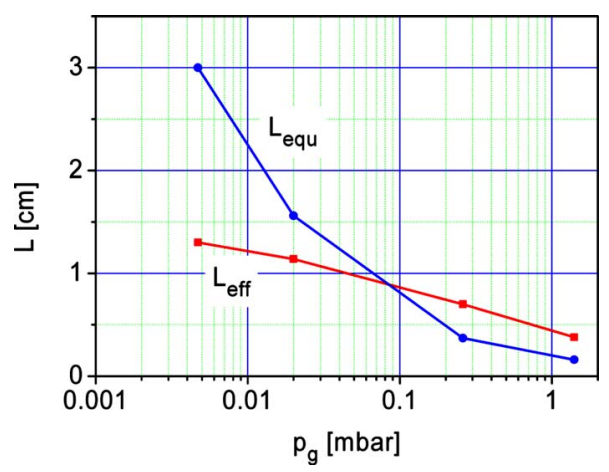

FIG. 2. (Color online) Comparison of the lengths $L_{\text {eff }}$ and $L_{\text {equ }}$ as defined in Fig. 1 plotted vs the feeding pressure $p_{g}$. Data are taken from earlier measurements (see Ref. 10).

$$
Q_{T}=C_{\text {mol }}\left(p_{\text {equ }}-p_{\text {orifice }}\right) \quad \text { with } \quad C_{\text {mol }}=\frac{2 \pi}{3} \bar{c} \frac{r^{3}}{L_{\text {equ }}},
$$

where $\bar{c}$ is the mean thermal speed and $r$ is the capillary radius. The pressure $p_{\text {orifice }}$ can be neglected. We refer to the data in Ref. 10, Table I, where $T=2600 \mathrm{~K}$. In the approximation $\alpha=1$, the amount of gas particles is doubled by the dissociation and the gas flow rate $Q_{T}=Q_{2600}$ is given by

$$
Q_{2600}=2 Q_{g} \frac{2600 \mathrm{~K}}{295 \mathrm{~K}},
$$

where $Q_{g}$ is the gas flow rate in the gas duct at room temperature. By applying Eqs. (1) and (2) we can enumerate $L_{\text {equ }}$ from the data of $p_{\text {equ }}$ and $Q_{g}$, as given in Ref. 10, Table I. $\left(Q_{g}\right.$ is termed $\dot{Q}_{g}$ in Ref. 10.)

Figure 2 shows $L_{\text {equ }}$ as well as $L_{\text {eff }}$ as a function of the feed pressure $p_{g}$. The model in Fig. 1 with $L_{\text {equ }}>L_{\text {eff }}$ is apparently valid for feed pressures $p_{g}<0.1 \mathrm{mbar}$. The hydrogen source of the hot capillary design is especially powerful in the low pressure range where the degree of dissociation is higher and the beam formation is more developed than those at high pressure. Both a high degree of dissociation and a slim beam contribute to a high ratio of the axial beam intensity to the gas load on the vacuum system. Operated in this pressure range, $p_{g}<0.1 \mathrm{mbar}$, the hottest part of the capillary should extend from a distance of about 3 to about $1 \mathrm{~cm}$ from the orifice. In the frame of our simple model, a temperature decrease next to the orifice is not detrimental. This conclusion was the reason why we tried radiative heating of the capillary, which presumably would not reach the temperature level and homogeneity of electron bombardment heating.

In designing a source with radiative heating, we started from our electron bombardment heated source retaining its basic features such as capillary, filament, thermal insulation, copper cap, and water cooling. However, the capillary was now to be heated by the thermal radiation from the resistively heated filament. Several different versions of the source were built at Jülich and tested with the aim of minimizing the heat loss by radiation through the thermal shields and the heat loss by conduction to the water-cooled socket. Mention should be made of a preliminary version where the capillary, its support, the filament, and the thermal shields 


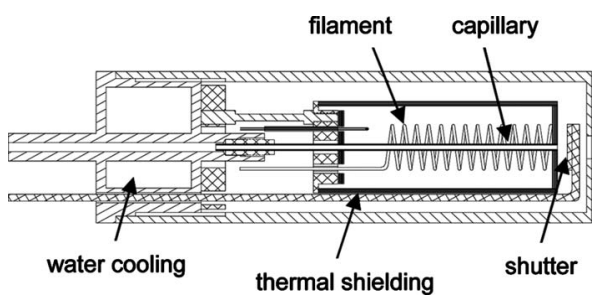

FIG. 3. Hydrogen atom source with radiative heating of the capillary.

were constructed as a unit which could be removed from the water-cooled body and the gas feed line in order to replace a broken filament. However, the filaments proved to be very reliable. The sealing between the removable capillary unit and the gas feed line, on the other hand, required careful handling. This feature was therefore abandoned in the final version in favor of simplicity. The preliminary and final versions were constructed several times and utilized in other laboratories. ${ }^{3-9}$ Finally Schuler revised the technical details of the source to optimize its fabrication. The present version of the source is sketched in Fig. 3. Capillary and filament are made of W. A typical capillary temperature of $2200 \mathrm{~K}$ is achieved at a filament power of around $160 \mathrm{~W}(I<14 \mathrm{~A}$, $U<13 \mathrm{~V})$. These conditions avoid any energetic particles. Dispensing with the high-voltage insulation, which is peculiar to electron bombardment heating, results in a much simpler design and a much higher reliability of the source. The filament is preferentially powered by a dc power supply in the constant current mode, which makes the source run stably and reproducibly.

\section{QUADRUPOLE MASS ANALYZER SYSTEM AND ITS CALIBRATION}

\section{A. Quadrupole mass analyzer system}

By switching from electron bombardment to radiative heating we decrease the capillary temperature range from a maximum of 2600 to typically $2200 \mathrm{~K}$. This is a significant temperature decrease with respect to dissociation requiring a highly sensitive hydrogen atom detection system. We therefore designed an improved analysis system incorporating as before a QMA for the detection of hydrogen atoms and molecules. The analysis system is shown schematically in Fig. 4. The QMA (Pfeiffer QMA 160, QMH 400-1, and QMG 422) is accommodated in a differentially pumped chamber whose base pressure is below the $\mathrm{x}$-ray limit of the ion gauge (2 $\left.\times 10^{-11} \mathrm{mbar}\right)$. The QMA is equipped with an axial beam ionizer for electron-impact ionization. The parameters of the ionizer are adjusted to optimize the sensitivity and resolution of masses 1 and 2 (filament to anode of $53 \mathrm{~V}$, anode to lens of $0 \mathrm{~V}$, anode to rod system entrance of $150 \mathrm{~V}$, and anode to rod system field axis of $18.25 \mathrm{~V}$ ). The ion current $I$ is detected by a Faraday cup.

Gas particles from the source chamber pass to the QMA chamber via a tube of $1 \mathrm{~mm}$ inner diameter and $10 \mathrm{~mm}$ length. The hydrogen atom source can be swiveled by $\pm 30^{\circ}$ about an axis through the orifice. This kind of setup was already used by Van Zyl and Gealy ${ }^{19}$ to record the angular distribution of the particles emanating from the orifice. The

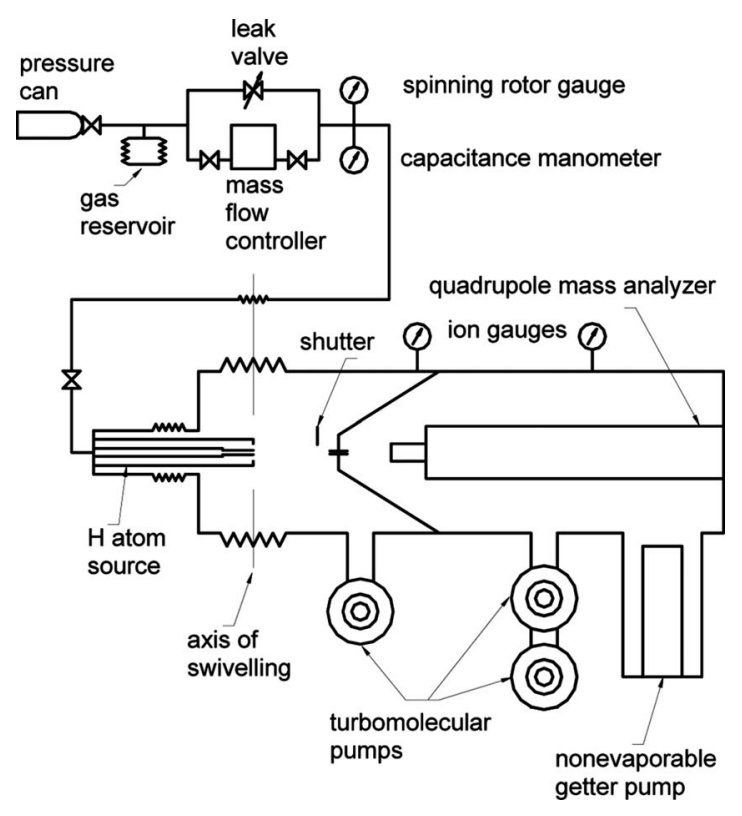

FIG. 4. Schematic of the apparatus used to determine the intensity of the hydrogen atom source.

source can be mechanically adjusted to bring the capillary into the line of sight of the pressure stage tube and the QMA ionizer. Hydrogen gas is admitted from a pressure can to a bellows-type gas reservoir and via either an UHV leak valve or a mass flow controller (Aera FC-901) to the source. The feed pressure is measured by a spinning rotor gauge or a capacitance manometer depending on the actual pressure. The pressure ranges are as follows: feed pressure $p_{g}=2$ $\times 10^{-3}, \ldots, 3.5$ mbars, pressure in the source chamber $p_{S}$ $=1.5 \times 10^{-8}, \ldots, 1.6 \times 10^{-5} \mathrm{mbar}$, and pressure in the QMA chamber $p_{Q}=<2 \times 10^{-11}, \ldots, 5 \times 10^{-9}$ mbar. The highest pressures correspond to a mass flow rate $Q=1$ SCCM (SCCM denotes cubic centimeter per minute at STP). This analysis system is superior to the former one in the sensitivity of the QMA, differential pumping performance and therefore in the background of the QMA signal, and width of the accessible polar angle range.

We previously determined the intensity of the source from three experimentally determined quantities: (1) the flow rate of the hydrogen feed gas, (2) the degree of dissociation indicating how much of the feed gas is dissociated, and (3) the angular distribution of the emitted hydrogen atoms. Quantity (1) was measured in absolute numbers and quantities (2) and (3) in relative numbers. The angular distribution was only measured at the highest capillary temperature $(2600 \mathrm{~K})$ and was assumed to be valid at all temperatures. In the present system, the improved signal-to-noise ratio and the low pressure in the QMA chamber make it feasible to register the angular distribution of the atoms as well as that of the molecules at arbitrary temperature. By integrating the angular distribution we obtain the total emitted flux of atoms and molecules which when combined have to balance the feed gas mass flow. This is the present route for achieving quantitative data of the intensity.

The full polar angle range $\left( \pm 30^{\circ}\right)$ of the analysis system is not illuminated by the standard source as shown in Fig. 3, 


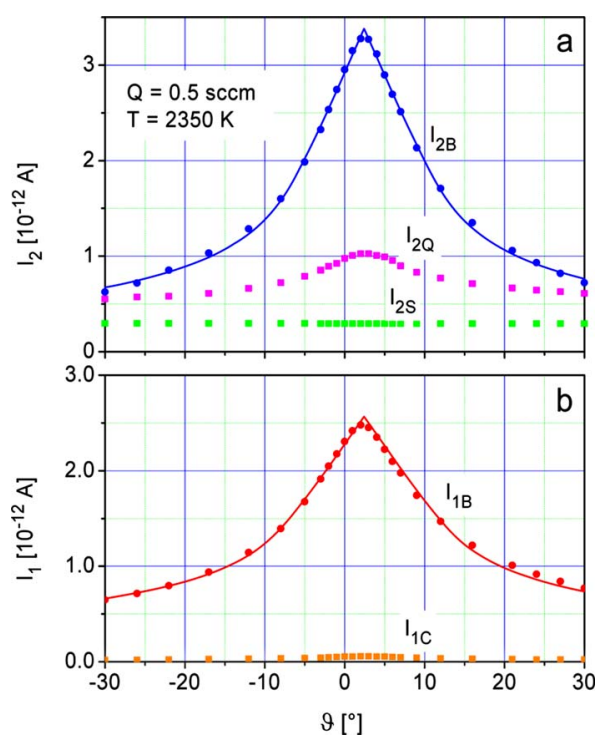

FIG. 5. (Color online) Angular distributions of the QMA currents of molecular ions (a) and atomic ions (b). The experimental data are fitted by an analytic function. The currents $I_{2 B}$ and $I_{1 B}$ represent the beam of molecules and atoms from the source. The currents $I_{2 S}$ and $I_{2 Q}$ originate from the gas in the source chamber and QMA chamber, respectively. $I_{1 C}$ is due to the cracking of molecules in the QMA ionizer. The deviation of the peaks of $I_{2 B}, I_{2 Q}$, and $I_{1 B}$ from $\vartheta=0^{\circ}$ is a systematic offset presumably arising from the shape of the vacuum system.

whose polar angle range is limited to around $20^{\circ}$ by the opening in the $\mathrm{Cu}$ cap. Therefore, we proceeded in two steps. (1) The QMA system is calibrated with a special source which had no structural material in front of the capillary orifice and whose angular distribution is accordingly bare of any shielding. (2) Thereafter a standard source is investigated in the calibrated QMA system.

We turn now to the calibration and describe the measurements on the standard source in the following.

\section{B. Calibration of the quadrupole mass analyzer system}

The special source is put into operation in a separate vacuum system where the capillary temperature is measured versus the heating power. The source is then mounted into the QMA system and the angular distributions of hydrogen atoms and molecules are recorded for different temperatures and mass flow rates. The mass flow is determined by the mass flow controller and the mass flow rate is given in SCCM (1 SCCM corresponds to a particle flux of 4.48 $\times 10^{17}$ particles $\left./ \mathrm{s}\right)$.

Figures 5(a) and 5(b) show an example of the angular distribution of molecules and atoms. The current $I_{2}$ of molecular ions [not shown in Fig. 5(a)] is the sum of the current $I_{2 B}$ due to the beam of molecules from the capillary and of the currents $I_{2 S}$ and $I_{2 Q}$ arising from the partial pressures of $\mathrm{H}_{2}$ in the source chamber and the QMA chamber, respectively. (The isotropic background gas in the source chamber contributes to the gas flow through the tube of the differential pumping stage into the QMA ionizer. $I_{2 S}$ is the corresponding current.) $I_{2 S}$ and $I_{2 Q}$ are proportional to the respective pressures and the appropriate factors were determined. $I_{2 S}$ and $I_{2 Q}$ can then be calculated from the experimental pressures and are subtracted from $I_{2}$ to get the proper beam signal $I_{2 B}$. The current $I_{1}$ of atomic ions [not shown in Fig. 5(b)] is the sum of the current $I_{1 B}$ from the beam of atoms emanating from the capillary and the current $I_{1 C}$ arising from the cracking of the molecules represented by the currents $I_{2 B}, I_{2 S}$, and $I_{2 Q}$. The appropriate cracking factors were determined. [In Fig. 5(b) $I_{1 C}$ is low as compared with $I_{1}$ due to the high temperature.]

The angular distribution of the molecules [Fig. 5(a)] is narrower than the angular distribution of the atoms [Fig. $5(\mathrm{~b})]$. The full widths at half maximum (FWHMs) are $19.1^{\circ}$ and $23.5^{\circ}$, respectively. The FWHM is smaller and the difference between molecules and atoms is more pronounced at low mass flow rate. At $Q=0.04 \mathrm{SCCM}(T=2270 \mathrm{~K})$ [as compared with $Q=0.5 \operatorname{SCCM}(T=2350 \mathrm{~K})$ for Fig. 5] the FWHMs are $5.5^{\circ}$ for molecules and $13.5^{\circ}$ for atoms. The corresponding lengths $L_{\text {eff }}$ of transparent flow in the capillary are $18 \mathrm{~mm}$ for molecules and $9 \mathrm{~mm}$ for atoms. This reflects the fact that atoms originate in the hot part of the capillary, which is closer to the orifice than to the socket, whereas molecules might pass through the capillary from a location near the socket to the orifice in a straight trajectory.

The experimental data of $I_{2 B}$ and $I_{1 B}$ are fitted by

$$
I_{i B}(\vartheta)=A_{i} j_{i}(\vartheta), \quad i=1,2,
$$

where $\vartheta$ is the polar angle, $A_{i}$ is the amplitude, and $j_{i}(\vartheta)$ is an analytic function describing the normalized angular distribution. ${ }^{10,11}$ Using an analytic function for the fit makes it feasible to extrapolate and integrate the angular distribution over the hemisphere in front of the capillary. (In the fitting process we had to allow for an angle-independent background signal which turned out not to be related to any of the above-mentioned currents.)

The currents $I_{i B}$ are proportional to the beam intensity $J_{i}$ (unit: atom or molecule/sr s) and we define the corresponding sensitivity factors $S_{i}$ by

$$
I_{i B}=f_{T} S_{i} J_{i}, \quad i=1,2 \quad \text { with } f_{T}=\sqrt{\frac{2000 \mathrm{~K}}{T}} .
$$

The square root term accounts for the time the particles spend in the ionizer. ${ }^{11,20}$ The reference temperature of 2000 $\mathrm{K}$ is arbitrary but fixed. Our sensitivity factors $S_{i}$ are related to this temperature.

We know the flux of molecules $\dot{N}_{g}$ into the capillary from the mass flow controller. This flux is balanced by the total fluxes of the atoms, $\dot{N}_{1}$, and of the molecules, $\dot{N}_{2}$, leaving the orifice.

$$
2 \dot{N}_{g}=\dot{N}_{1}+2 \dot{N}_{2} \text {. }
$$

From $\dot{N}_{i}=\int_{2 \pi} J_{i}(\vartheta) d \omega$ and Eqs. (3)-(5) we obtain

$$
\begin{aligned}
& \dot{N}_{i}=\int_{2 \pi} \frac{1}{f_{T} S_{i}} A_{i} j_{i}(\vartheta) d \omega \\
& =\frac{1}{f_{T} S_{i}} A_{i} \Omega_{i} \text { where } \Omega_{i}=\int_{2 \pi} j_{i}(\vartheta) d \omega,
\end{aligned}
$$




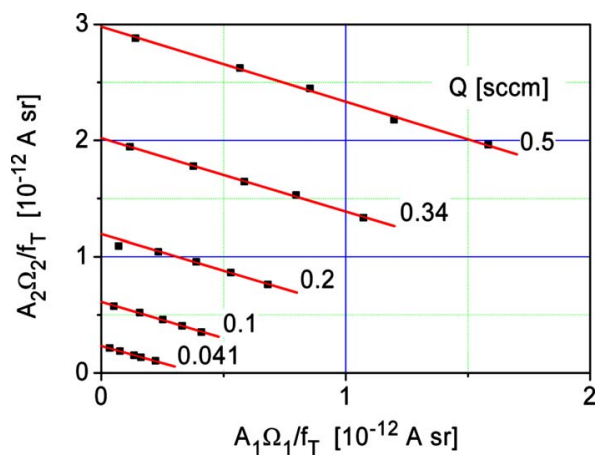

FIG. 6. (Color online) The integrated QMA signal $A_{2} \Omega_{2} / f_{T}$ for hydrogen molecules plotted vs the equivalent signal $A_{1} \Omega_{1} / f_{T}$ for hydrogen atoms. The abscissa is proportional to the total atomic flux and the ordinate is proportional to the total molecular flux. The atom flux increases at the expense of the molecular flux.

$$
\begin{aligned}
& 2 \dot{N}_{g}=\frac{1}{f_{T} S_{1}} A_{1} \Omega_{1}+\frac{2}{f_{T} S_{2}} A_{2} \Omega_{2}, \\
& \frac{1}{f_{T}} A_{2} \Omega_{2}=S_{2} \dot{N}_{g}-\frac{S_{2}}{2 S_{1}} \frac{1}{f_{T}} A_{1} \Omega_{1} .
\end{aligned}
$$

In a series of measurements we applied different mass flow rates by means of the mass flow controller and varied the temperature from 1870 to around $2300 \mathrm{~K}$ for each mass flow setting. The measured angular distributions were fitted to obtain $A_{i}$ and $j_{i}(\vartheta)$. By integrating the latter we obtain $\Omega_{i}$. Figure 6 shows $A_{2} \Omega_{2} / f_{T}$ versus $A_{1} \Omega_{1} / f_{T}$ for this series of measurements. Note that the ordinate is proportional to the molecular flux out of the capillary and that the abscissa is proportional to the corresponding atomic flux. The temperature increases along every data set from left to right. Figure 6 describes the increase in the atomic flux at the expense of the molecular flux.

The data of Fig. 6 are fitted by straight lines

$$
\frac{1}{f_{T}} A_{2} \Omega_{2}=a-b \frac{1}{f_{T}} A_{1} \Omega_{1} .
$$

The coefficients $a$ and $b$ are plotted in Fig. 7 versus the mass flow settings used in this series of measurements. The coefficient $b$ is constant whereas $a$ is nicely proportional to the mass flow. The slope of $a$ is equal to $S_{2}$, as can be seen by comparing Eqs. (9) and (10). Replacing 1 SCCM by 4.48 $\times 10^{17}$ particles/s we obtain

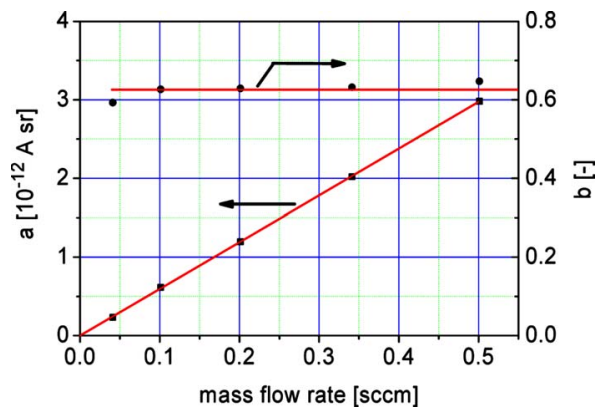

FIG. 7. (Color online) The absolute term $a$ and the slope $b$ of the fitting lines in Fig. 6.



FIG. 8. (Color online) The on-axis flux density $\varphi_{1}$ of hydrogen atoms on a substrate at $d=6 \mathrm{~cm}$ distance plotted vs the heating power. The parameter is the mass flow rate $Q$. The gas is admitted to the source via a mass flow controller.

$$
S_{2}=1.33 \times 10^{-29} \mathrm{~A} /(\text { molecule/sr s }) .
$$

Likewise we find from comparing Eqs. (9) and (10) $S_{1}$ $=S_{2} /(2 b)$ and

$$
S_{1}=1.06 \times 10^{-29} \mathrm{~A} /(\text { atom } / \mathrm{sr} \mathrm{s}) .
$$

It is interesting to note that $S_{1}$ is smaller by a factor of about $1 / 20$ as compared with the earlier analysis system. ${ }^{11}$ Nevertheless, the signal-to-noise ratio is much higher and the background pressures are much lower in the present system, resulting in a much lower limit of particle detection.

\section{CHARACTERIZATION OF THE SOURCE}

Knowledge of the sensitivity factors of the QMA system enables us to determine the intensity of the standard source (Fig. 3) for various settings of gas flow and heating power. In a preparatory step the source is put into operation in a separate vacuum system where the capillary temperature is measured as a function of the heating power. After transfer into the QMA system, the source is adjusted and maintained at the polar angle of $\vartheta=0^{\circ}$ to measure the on-axis intensity.

Hydrogen gas is fed to the source by the mass flow controller when higher flow rates are required or via the leak valve in the case of lower flow rates. Higher mass flow rates extend from 0.02 to 1 SCCM. With the mass flow rate set, we vary the heating power and accordingly the capillary temperature. The QMA signal due to the atoms and molecules from the source is identified as the signal step when a shutter at the pressure stage is opened. As described above for the special source, the raw signal $I_{2}$ is corrected for the currents $I_{2 S}$ and $I_{2 Q}$ to obtain the proper beam signal $I_{2 B}$ and the raw signal $I_{1}$ is corrected for $I_{1 C}$ to obtain $I_{1 B}$. By the use of Eq. (4) and the sensitivity factors $S_{1}$ and $S_{2}$, we obtain the beam intensities $J_{i}$ in units of atoms/sr s or molecules/sr s. More illustrative than the intensity $J_{i}$ is the flux density $\varphi_{i}$ onto a substrate, which is evaluated by

$$
\varphi_{i}=\frac{J_{i} \cdot \mathrm{sr}}{d^{2}} .
$$

In Fig. 8 we plot the on-axis atom flux density $\varphi_{1}$ onto a substrate at a distance of $6 \mathrm{~cm}$ from the capillary orifice. An impingement rate corresponding to a monolayer per second is achieved at a mass flow rate of 0.1 SCCM and a heating 


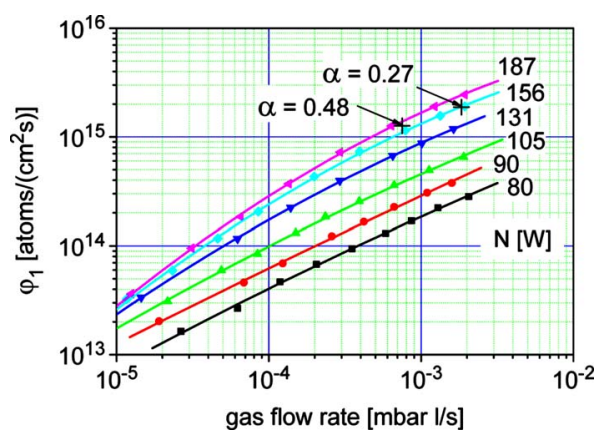

FIG. 9. (Color online) The on-axis flux density $\varphi_{1}$ of hydrogen atoms on a substrate at $d=6 \mathrm{~cm}$ distance plotted vs the gas flow rate $Q_{g}$. The parameter is the heating power $N$. The power steps from 80 to $187 \mathrm{~W}$ correspond to temperatures of $1840,1900,1980,2100,2200$, and $2300 \mathrm{~K}$, respectively. Values of the degree of dissociation $\alpha$ are indicated. The gas is admitted to the source via a leak valve.

power of $160 \mathrm{~W}$. Higher impingement rates require higher mass flow rates.

Mass flow rates lower than 0.02 SCCM can only be admitted via the leak valve which, however, cannot be set to a certain mass flow rate. Therefore, we preset the heating power as the parameter and vary the gas flow by the leak valve. After reaching stationary pressures, we measure the gas feed pressure $p_{g}$ and calculate the gas flow rate $Q_{g}$ by

$$
Q_{g}=C_{g} p_{g} .
$$

$C_{g}$ is the flow conductance of the hot source, which can be evaluated from the previous mass flow controller operation, where we set the mass flow rate $Q$ (SCCM) and measure $p_{g}$. We convert the mass flow rate $Q$ into the gas flow rate $Q_{g}$ at room temperature $\left(1 \mathrm{SCCM}=1.82 \times 10^{-2} \mathrm{mbar} 1 / \mathrm{s}\right.$ at 295 K) and evaluate $C_{g}$ by Eq. (12).

The atom flux density $\varphi_{1}$ as measured with gas feed by the leak valve is depicted in Fig. 9. Flux densities down to the order of $10^{13}$ atoms $/ \mathrm{cm}^{2} \mathrm{~s}$ can be set in a controlled manner. The fitting lines for high heating power merge at low gas flow rates. This is indicative of the fact that the degree of dissociation $\alpha$ approaches 1 . Two values of $\alpha$ are indicated in the figure. These data are derived from measurements with the special source since in this work the determination of $\alpha$ requires the knowledge of the angular distribution. The variation of $\alpha$ with temperature was presented in an earlier publication $^{11}$ (See Table I and Fig. 6. There we find $\alpha=0.3$ instead of 0.27 for $Q_{g}=1.69 \times 10^{-3} \mathrm{mbar} 1 / \mathrm{s}$ and $T$ $=2200 \mathrm{~K}$.)

The data in Figs. 8 and 9 are fitted by analytic functions. These are used to set up a contour plot of the on-axis atom flux density as a function of the mass flow rate and the heating power, see Fig. 10. The flux density covers a range of two orders of magnitude and extends up to some $10^{15}$ atoms $/ \mathrm{cm}^{2} \mathrm{~s}$.

\section{CONCLUSION}

Our earlier measurements on a hydrogen atom source incorporating a hot capillary heated by electron bombardment are described by a simple model. We conclude from this model that heating of the capillary by thermal radiation should work just as effectively if the mass flow rate of the

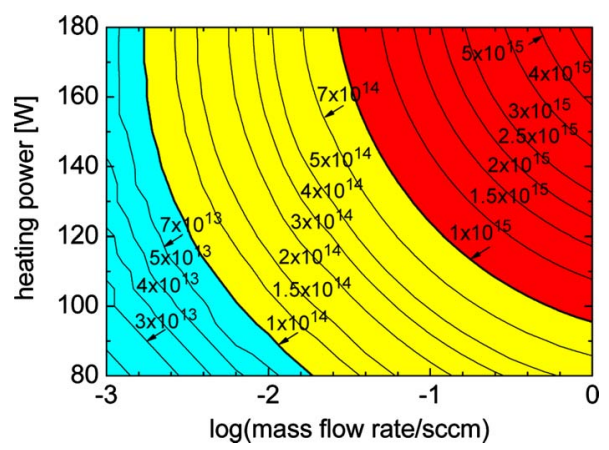

FIG. 10. (Color online) Contour plot of the on-axis flux density $\varphi_{1}$ of hydrogen atoms on a substrate at $d=6 \mathrm{~cm}$ distance. The experimental parameters are the mass flow rate and the heating power.

feeding hydrogen gas is in a lower range. Switching from electronic to radiative heating eliminates the need for high voltage and its insulation, which significantly simplifies the design of a source as well as its power equipment. Our final design for a radiatively heated source proves to be much more reliable than its electronically heated predecessor.

In order to determine the intensity of the source a special QMA system is designed and built. The sensitivity of the system is evaluated for atoms as well as for molecules and the on-axis intensity of the source is determined. The intensity of the source can be adjusted by the hydrogen mass flow rate and the heating power. A contour plot as a function of these parameters is set up showing a variation of the on-axis intensity over two orders of magnitude ranging from some $10^{13}$ to some $10^{15}$ atoms $/ \mathrm{cm}^{2} \mathrm{~s}$ if an orifice-to-substrate distance of $6 \mathrm{~cm}$ is assumed. In conclusion, a radiatively heated hot capillary source is felt to be superior to an electronically heated source if it is intended to achieve hydrogen atom intensities in the aforementioned range.

\section{ACKNOWLEDGMENTS}

Many helpful discussions with U. Littmark are gratefully acknowledged as well as the comments on the manuscript by him and J. Carter-Sigglow. One of us (K.G.T.) is grateful to R. Merkel for supporting this work.

${ }^{1}$ A. Crossley, C. J. Sofield, S. Sugden, R. Clampitt, and C. Bradley, Vacuum 46, 667 (1995).

${ }^{2}$ K. Yamaguchi, Zh. Qin, H. Nagano, M. Kobayashi, A. Yoshikawa, and K. Takahashi, Jpn. J. Appl. Phys., Part 2 36, L1367 (1997).

${ }^{3}$ C. Tölkes, R. David, K. G. Tschersich, G. Comsa, and P. Zeppenfeld, Europhys. Lett. 46, 589 (1999).

${ }^{4}$ M. Eremtchenko, F. S. Tautz, R. Öttking, and J. A. Schaefer, Surf. Sci. 600, 3446 (2006).

${ }^{5}$ L. Hornekær, Z. Sljivancanin, W. Xu, R. Otero, E. Rauls, I. Stensgaard, E. Lægsgaard, B. Hammer, and F. Besenbacher, Phys. Rev. Lett. 96, 156104 (2006).

${ }^{6}$ B. Mildner, E. Hasselbrink, and D. Diesing, Chem. Phys. Lett. 432, 133 (2006).

${ }^{7}$ S. Baouche, G. Gamborg, V. V. Petrunin, A. C. Luntz, and A. Baurichter, J. Chem. Phys. 125, 084712 (2006).

${ }^{8}$ R. J. Westerwaal, M. Slaman, C. P. Broedersz, D. M. Borsa, B. Dam, R. Griessen, A. Borgschulte, W. Lohstroh, B. Kooi, G. ten Brink, K. G. Tschersich, and H. P. Fleischhauer, J. Appl. Phys. 100, 063518 (2006).

${ }^{9}$ K. G. Tschersich, U. Littmark, and W. Beyer, Thin Solid Films 515, 464 (2006).

${ }^{10}$ K. G. Tschersich and V. von Bonin, J. Appl. Phys. 84, 4065 (1998).

${ }^{11}$ K. G. Tschersich, J. Appl. Phys. 87, 2565 (2000). 
${ }^{12}$ J.-Y. Kim and J. Lee, J. Chem. Phys. 113, 2856 (2000).

${ }^{13}$ U. Bischler and E. Bertel, J. Vac. Sci. Technol. A 11, 458 (1993).

${ }^{14}$ A. Horn, Th. Kammler, and M. Kappel, Patent No. DE 19757851 C1, 1999.

${ }^{15}$ Th. Zecho, B. D. Brandner, J. Biener, and J. Küppers, J. Phys. Chem. B 105, 3502 (2001)

${ }^{16}$ Th. Schwarz-Selinger, A. von Keudell, and W. Jacob, J. Vac. Sci. Technol.
A 18, 995 (2000).

${ }^{17}$ S. Dushman, Scientific Foundations of Vacuum Technique (Wiley, New York, 1962), p. 58.

${ }^{18}$ J. O'Hanlon, A User's Guide to Vacuum Technology (Wiley, New York, 1989), p. 22.

${ }^{19}$ B. Van Zyl and M. W. Gealy, Rev. Sci. Instrum. 57, 359 (1986).

${ }^{20}$ J. N. Smith and W. L. Fite, J. Chem. Phys. 37, 898 (1962). 